\title{
Sentencias por culpa patronal en accidentes de trabajo en Colombia. Una mirada desde la Seguridad y la Salud en el Trabajo*
}

[Artículos]

\author{
Nicolás Calderón Grisales** \\ Lina María Trujillo Flórez ${ }^{* * *}$ \\ Liliana Parra Osorio ${ }^{* * * *}$
}

Fecha de recepción: 4 de marzo de 2021

Fecha de aprobación: 18 de mayo de 2021

Citar como

Calderón Grisales, N., Trujillo Flórez, L. M. y Parra Osorio, L. (2021). Sentencias por culpa patronal en accidentes de trabajo en Colombia. Una mirada desde la seguridad y la salud en el trabajo. Via Inveniendi Et Iudicandi, 16(2).

https://doi.org/10.15332/19090528.6780

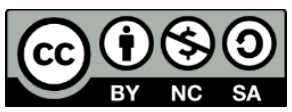

\section{Resumen}

La culpa patronal por accidente de trabajo es un concepto de origen jurisprudencial y doctrinal, sus fuentes son la responsabilidad civil del

\footnotetext{
${ }^{*}$ El artículo es producto del macroproyecto de investigación: "La legislación en la protección de los peligros, riesgos laborales y la salud laboral en Colombia", del grupo de investigación Estudios Interdisciplinarios DESC y Mundo del Trabajo, del Centro de Investigaciones Socio jurídicas de la Facultad de Derecho de la Universidad Libre, Bogotá.

${ }^{* *}$ Abogado, auxiliar administrativo grado 19, Secretaría Distrital de Gobierno. Correo electrónico: ncalderon-12@hotmail.com. ORCID: https://orcid.org/0000-0001-6767-2256

${ }^{* * *}$ Abogada, analista jurídica, Masivo Capital S. A. S. Correo electrónico: linamtrujillof@gmail.com. ORCID: https://orcid.org/0000-0002-6021-8015

**** Doctora en Ciencias de la Salud en el Trabajo de la Universidad Guadalajara de México, magíster en Salud Ocupacional de la Universidad del Valle. Docente investigadora del Centro de Investigaciones Socio jurídicas de la Facultad de Derecho de la Universidad Libre, Bogotá. Correo electrónico: liliana.parrao@unilibre.edu.co. ORCID: https://orcid.org/0000-0002-4128-4068
}

Via Inveniendi Et Iudicandi

e-ISSN: 1909-0528 | DOI: https://doi.org/10.15332/19090528

Vol. 16 N.o 2 | julio-diciembre del 2021 
artículo 1604 del Código Civil colombiano y la culpa del empleador del artículo 216 del Código Sustantivo del Trabajo. El objetivo es explorar las determinaciones de la Corte Suprema de Justicia, Sala de Casación Laboral sobre accidentes de trabajo con culpa patronal frente a los estándares mínimos en gestión de peligros y riesgos del Sistema de Gestión de Seguridad y Salud en el Trabajo. Se realizó una revisión documental de sentencias condenatorias por accidente de trabajo con culpa patronal. Los criterios: condena por culpa patronal en accidente de trabajo, 2019-2020, Sector Privado, Sala de Casación Laboral. Una matriz en Excel (anexo 1) fue instrumento de recolección, se seleccionaron 76 sentencias. Se hizo un análisis de tipo cualitativo en tres fases: descripción, categorización e interpretación. Los resultados arrojaron en consideraciones ratio decidendi el incumplimiento del empleador en normas en seguridad y salud en el trabajo como suministro de elementos de protección personal, diligencia y cuidado, conducta omisiva, supervisión de normas, protocolos de seguridad, capacitación y entrenamiento, herramientas y equipos seguros. En conclusión, hubo conducta negligente y descuidada, lo cual mostró que la mayoría de estos accidentes de trabajo, severos y mortales, ocurren debido a que el empleador no tuvo en cuenta un enfoque preventivo desde el control y la gestión de peligros y riesgos laborales.

Palabras clave: sentencias, accidente de trabajo, culpa patronal, seguridad y salud en el trabajo.

\section{Judgments for employer's negligence in occupational accidents in Colombia. An occupational health and safety perspective}

\section{Abstract}

Employer's negligence for occupational accidents is a concept of jurisprudential and doctrinal origin, its sources are the civil liability of article 1604 of the Colombian Civil Code and the employer's negligence of article 216 of the Substantive Labor Code. The objective is to explore 
the decisions of the Supreme Court of Justice, Labor Cassation Chamber on occupational accidents with employer's negligence in the face of the minimum standards in hazards and risks management of the Occupational Health and Safety Management System. A documentary review of guilty verdicts for occupational accidents due to employer's negligence was carried out. Criteria: guilty verdicts due to employer's negligence in occupational accident, 2019-2020, Private Sector, Labor Cassation Chamber. An Excel matrix (Annex 1) was used as a collection instrument, 76 judgments were selected. A qualitative analysis was made in three phases: description, categorization and interpretation. The results showed in ratio decidendi considerations the employer failure to comply with the Occupational Safety and Health standards such as provision of personal protective equipment, diligence and care, omissive conduct, supervision of standards, safety protocols, training, safe tools and equipment. In conclusion, there was negligent and careless conduct, which showed that most of these severe and fatal occupational accidents occur because the employer did not take into account a preventive approach from the control and occupational hazards and risks management.

Keywords: judgments, occupational accident, employer's negligence, Occupational Safety and Health.

\section{Introducción}

La culpa patronal surge como una solución a la desprotección de los trabajadores, debido a la aplicación de las teorías de la responsabilidad anteriores a esta, como es el caso de i) la teoría de la responsabilidad civil extracontractual, ii) la teoría de la responsabilidad civil contractual y iii) la teoría de la inversión de la carga de prueba. La teoría de la culpa patronal indica que es deber del empleador responder cuando su trabajador ha sufrido un accidente de trabajo, solo si el primero no ha verificado que las condiciones en las que está trabajando el segundo son seguras. En materia de culpa patronal esta investigación tendrá como referente la teoría de 
riesgo creado, la cual implica que el empleador es responsable si no ha verificado que las condiciones en las que está el trabajador son adecuadas para su salud y seguridad. Es por esto, que esta teoría ha sido tenida en cuenta, ya que implica el cumplimiento por parte del empleador de los estándares mínimos en gestión de peligros y riesgos en seguridad y salud en el trabajo.

Así mismo, por medio de leyes y actos administrativos se ha buscado regular la Seguridad y Salud en Trabajo con el fin de reducir la accidentalidad laboral, tales como Ley 9 de 1979, Resolución 2400 de 1979, Resolución 1016 de 1989, Decreto 1295 de 1994, Resolución 1407 de 2007, Decreto 1072 de 2015, Ley 1562 de 2012, Decreto 1072 de 2019 de 2015 y Resolución 0312 de 2019.

A pesar de la regulación anterior, en el 2020, en Colombia se presentaron 450110 accidentes de trabajo calificados y 454 muertes reportadas por presuntos accidentes de trabajo (Ministerio del Trabajo, 2021). El Ministerio del Trabajo apoya al sistema general de riesgos laborales para la reducción de la accidentalidad; esto conduce al interrogante de si la normatividad reciente en materia de seguridad y salud en el trabajo ha sido revisada en los casos que llegan a la Corte Suprema de Justicia. Por tanto, el objetivo es explorar las determinaciones de la Corte Suprema de Justicia, Sala de Casación Laboral sobre accidentes de trabajo con culpa patronal frente a los estándares mínimos en gestión de peligros y riesgos del Sistema de Gestión de Seguridad y Salud en el Trabajo entre 2019 y 2020.

\section{Problema de investigación}

¿Qué ha determinado la Corte Suprema de Justicia en las sentencias de casación laboral frente al accidente de trabajo en los cuales se demuestra la culpa patronal en relación con los estándares mínimos en gestión de

Via Inveniendi Et Iudicandi

e-ISSN: 1909-0528 | DOI: https://doi.org/10.15332/19090528

Vol. 16 N.० 2 | julio-diciembre del 2021 
peligros y riesgos del Sistema de Gestión de Seguridad y Salud en el Trabajo entre 2019 y 2020 ?

\section{Métodos}

El enfoque de esta investigación es cualitativo, “cuyas características son las interpretaciones de los datos, tiene un proceso inductivo y posee las bondades de riqueza interpretativa y contextualiza el fenómeno estudiado" (Sampieri, Fernández, Baptista, 2006, p. 2). La recolección de los datos consiste en obtener las perspectivas, explorar las determinaciones, punto de vista y de decisión en las sentencias a la luz de los estándares mínimos en seguridad y salud en el trabajo, específicamente en gestión de peligros y riesgos incluidos en la Resolución 0312 de 2019.

También, para este proceso, se realizó una revisión documental, en la que la unidad de análisis son todas las sentencias de culpa patronal en accidentes de trabajo del sector privado, puntualmente las condenatorias por la Corte Suprema de Justicia en la Sala de Casación Laboral. Además, se utilizaron técnicas de revisión sistemática exploratoria, mediante un protocolo de búsqueda con criterios de selección como condena por culpa patronal en accidente de trabajo (2019 y 2020), sector privado y Sala de Casación Laboral. Se usó una matriz para la recolección de información, en la que se sistematizaron los contenidos de las sentencias con las siguientes variables: número de la sentencia, tipo de sentencia, año, magistrado ponente, corte o tribunal, hechos, problema jurídico, consideraciones más relevantes y resuelve. También, las variables concernientes a seguridad y salud en el trabajo a la luz de la Resolución 0312 de 2019, como los estándares mínimos señalados en la gestión de los peligros y riesgos del Sistema de Gestión para la Seguridad y la Salud en el Trabajo, como las medidas de prevención y el control frente a los peligros y riesgos identificados (eliminación, sustitución, controles de ingeniería, controles

Via Inveniendi Et Iudicandi

e-ISSN: 1909-0528 | DOI: https://doi.org/10.15332/19090528

Vol. 16 N.० 2 | julio-diciembre del 2021 
administrativos y elementos de protección personal), aplicación de medidas de prevención y control por parte de los trabajadores, procedimientos e instructivos internos de seguridad y salud en el trabajo, inspecciones a instalaciones, maquinaria o equipos, mantenimiento periódico de las instalaciones, equipos, máquinas y herramientas, entrega de los elementos de protección personal y capacitación para el uso adecuado y método de investigación del accidente de trabajo (enfoque multicausal), causas inmediatas (actos inseguros y condiciones inseguras), causas básicas (factores personales y factores de trabajo) y factores de control (Resolución 0312, 2019, pp. 20-21).

Y para la exploración, comprensión y análisis de los contenidos de las sentencias se usó el análisis cualitativo con el método fenomenológico: 1) descripción: caracterización de las sentencias; 2) tematización o reducción; e 3) interpretación. Para llevar a cabo esto, se realizó la lectura y relectura de los contenidos de las sentencias, este proceso se hace en consenso de los investigadores, definen una línea base, la estandarización para la definición de las unidades de análisis o segmentación del contenido de las sentencias de culpa patronal por accidente de trabajo.

Luego, se inició nuevamente una relectura de las unidades de análisis (segmentos seleccionados que proporcionan un significado de los datos o contenidos de las sentencias), de acuerdo con la pregunta y los objetivos de la investigación. Y se organizaron por unidades similares, agrupación de códigos y se denominaron las categorías.

Finalmente, se llegó a la interpretación, mediante una agrupación global con la superfamilia que permite ver el todo, sus articulaciones y así generar aproximación explicativa a través de un diagrama que permita una aproximación explicativa del fenómeno estudiado. 


\section{Resultados}

\section{Capítulo 1. Descripción y categorización de los contenidos de las}

sentencias

Se inició un proceso de revisión de sentencias, encontrándose 169 por culpa patronal. Al hacer las selecciones por criterios de inclusión hubo que excluir 29 de ellas, porque no eran por accidentes de trabajo, quedando 140 sentencias. De estas, en sus revisiones, se encontró que era necesario excluir las de tutela por violación al debido proceso, permanenciendo 123, de las cuales fueron retiradas 11, porque su problema jurídico no hacía referencia a la ocurrencia de un accidente de trabajo por culpa patronal. Finalmente, quedaron seleccionadas por criterios de inclusión en el estudio 76 de ellas.

Tabla 1. Sentencias con culpa patronal por accidente de trabajo de 2019 y 2020

\begin{tabular}{|c|c|c|c|c|c|}
\hline Año & $\begin{array}{c}\mathrm{N} .^{\circ} \text { de } \\
\text { sentencia }\end{array}$ & Magistrado ponente & Año & $\begin{array}{c}\mathrm{N} .{ }^{\circ} \text { de } \\
\text { sentencia }\end{array}$ & Magistrado ponente \\
\hline 2019 & $\begin{array}{l}\text { SL5252- } \\
2019\end{array}$ & Carlos Arturo Guarín & 2019 & SL786-2019 & Jimena Isabel Godoy \\
\hline 2019 & $\begin{array}{l}\text { SL4811- } \\
2019\end{array}$ & Ernesto Forero Vargas & 2019 & SL795-2019 & Omar de Jesús Restrepo \\
\hline 2019 & $\begin{array}{l}\text { SL4653- } \\
2019\end{array}$ & Ernesto Forero Vargas & 2019 & SL651-2019 & Cecilia Margarita Durán \\
\hline 2019 & $\begin{array}{l}\text { SL4849- } \\
2019\end{array}$ & $\begin{array}{c}\text { Giovanni Francisco } \\
\text { Rodríguez }\end{array}$ & 2019 & SL583-2019 & Jorge Prada Sánchez \\
\hline 2019 & $\begin{array}{l}\text { SL4455- } \\
2019\end{array}$ & Jorge Prada Sánchez & 2019 & SL566-2019 & Jimena Isabel Godoy \\
\hline 2019 & $\begin{array}{l}\text { SL4473- } \\
2019\end{array}$ & Omar De Jesús Restrepo & 2019 & SL445-2019 & Ernesto Forero Vargas \\
\hline 2019 & $\begin{array}{l}\text { SL4965- } \\
2019\end{array}$ & Ana María Muñoz Segura & 2019 & SL354-2019 & Ernesto Forero Vargas \\
\hline 2019 & $\begin{array}{l}\text { SL4570- } \\
2019\end{array}$ & Clara Cecilia Dueñas & 2020 & $\begin{array}{l}\text { SL3048- } \\
2020\end{array}$ & Dolly Amparo Caguasango \\
\hline 2019 & $\begin{array}{l}\text { SL3897- } \\
2019\end{array}$ & Cecilia Margarita Durán & 2020 & $\begin{array}{l}\text { SL2906- } \\
2020\end{array}$ & Omar de Jesús Restrepo \\
\hline 2019 & $\begin{array}{l}\text { SL3915- } \\
2019\end{array}$ & Santander Rafael Brito & 2020 & $\begin{array}{l}\text { SL2653- } \\
2020\end{array}$ & Omar de Jesús Restrepo \\
\hline
\end{tabular}

Via Inveniendi Et Iudicandi

e-ISSN: 1909-0528 | DOI: https://doi.org/10.15332/19090528

Vol. 16 N.o 2 | julio-diciembre del 2021 


\begin{tabular}{|c|c|c|c|c|c|}
\hline Año & $\begin{array}{c}\mathrm{N} .{ }^{\circ} \mathrm{de} \\
\text { sentencia }\end{array}$ & Magistrado ponente & Año & $\begin{array}{c}\mathrm{N} .{ }^{\circ} \mathrm{de} \\
\text { sentencia }\end{array}$ & Magistrado ponente \\
\hline 2019 & $\begin{array}{l}\text { SL3664- } \\
2019\end{array}$ & Dolly Amparo Caguasango & 2020 & $\begin{array}{l}\text { SL2629- } \\
2020\end{array}$ & Santander Rafael Brito \\
\hline 2019 & $\begin{array}{l}\text { SL3693- } \\
2019\end{array}$ & Ana María Muñoz Segura & 2020 & $\begin{array}{l}\text { SL2388- } \\
2020\end{array}$ & Ana María Muñoz Segura \\
\hline 2019 & $\begin{array}{l}\text { SL3536- } \\
2019\end{array}$ & Donald José Dix Ponnefz & 2020 & $\begin{array}{c}\text { SL2335- } \\
2020\end{array}$ & Fernando Castillo Cadena \\
\hline 2019 & $\begin{array}{l}\text { SL3588- } \\
2019\end{array}$ & $\begin{array}{c}\text { Giovanni Francisco } \\
\text { Rodríguez }\end{array}$ & 2020 & $\begin{array}{l}\text { SL2491- } \\
2020\end{array}$ & Santander Rafael Brito \\
\hline 2019 & $\begin{array}{c}\text { SL3672- } \\
2019\end{array}$ & Jimena Isabel Godoy & 2020 & $\begin{array}{c}\text { SL1906- } \\
2020\end{array}$ & Omar de Jesús Restrepo \\
\hline 2019 & $\begin{array}{l}\text { SL3537- } \\
2019\end{array}$ & Carlos Arturo Guarín & 2020 & $\begin{array}{l}\text { SL1565- } \\
2020\end{array}$ & Martín Emilio Beltrán \\
\hline 2019 & $\begin{array}{c}\text { SL3214- } \\
2019\end{array}$ & Dolly Amparo Caguasango & 2020 & $\begin{array}{c}\text { SL1598- } \\
2020\end{array}$ & Jimena Isabel Godoy \\
\hline 2019 & $\begin{array}{c}\text { SL3498- } \\
2019\end{array}$ & Ernesto Forero Vargas & 2020 & $\begin{array}{c}\text { SL1743- } \\
2020\end{array}$ & Ana María Muñoz Segura \\
\hline 2019 & $\begin{array}{c}\text { SL2777- } \\
2019\end{array}$ & Jimena Isabel Godoy & 2020 & $\begin{array}{c}\text { SL1558- } \\
2020\end{array}$ & Martín Emilio Beltrán \\
\hline 2019 & $\begin{array}{c}\text { SL2782- } \\
2019\end{array}$ & Jimena Isabel Godoy & 2020 & $\begin{array}{c}\text { SL1608- } \\
2020\end{array}$ & Donald José Dix Ponnefz \\
\hline 2019 & $\begin{array}{c}\text { SL2831- } \\
2019\end{array}$ & Donald José Dix Ponnefz & 2020 & $\begin{array}{c}\text { SL3156- } \\
2020\end{array}$ & Dolly Amparo Caguasango \\
\hline 2019 & $\begin{array}{c}\text { SL2680- } \\
2019\end{array}$ & Jimena Isabel Godoy & 2020 & $\begin{array}{c}\text { SL1388- } \\
2020\end{array}$ & Dolly Amparo Caguasango \\
\hline 2019 & $\begin{array}{c}\text { SL2689- } \\
2019\end{array}$ & Cecilia Margarita Durán & 2020 & $\begin{array}{c}\text { SL1438- } \\
2020\end{array}$ & Jorge Prada Sánchez \\
\hline 2019 & $\begin{array}{c}\text { SL2783- } \\
2019\end{array}$ & Omar de Jesús Restrepo & 2020 & $\begin{array}{c}\text { SL1426- } \\
2020\end{array}$ & Jorge Prada Sánchez \\
\hline 2019 & $\begin{array}{l}\text { SL2426- } \\
2019\end{array}$ & Jorge Prada Sánchez & 2020 & $\begin{array}{l}\text { SL1465- } \\
2020\end{array}$ & Carlos Arturo Guarín \\
\hline 2019 & $\begin{array}{c}\text { SL1848- } \\
2019\end{array}$ & Martín Emilio Beltrán & 2020 & $\begin{array}{l}\text { SL1336- } \\
2020\end{array}$ & Martín Emilio Beltrán \\
\hline 2019 & $\begin{array}{l}\text { SL1999- } \\
2019\end{array}$ & Donald José Dix Ponnefz & 2020 & $\begin{array}{l}\text { SL1083- } \\
2020\end{array}$ & Martín Emilio Beltrán \\
\hline 2019 & $\begin{array}{c}\text { SL1911- } \\
2019\end{array}$ & Clara Cecilia Dueñas & 2020 & $\begin{array}{l}\text { SL1187- } \\
2020\end{array}$ & Ana María Muñoz Segura \\
\hline 2019 & $\begin{array}{l}\text { SL2206- } \\
2019\end{array}$ & Jorge Luis Quiroz Alemán & 2020 & SL696-2020 & Jimena Isabel Godoy \\
\hline 2019 & $\begin{array}{l}\text { SL1581- } \\
2019\end{array}$ & Jimena Isabel Godoy & 2020 & SL573-2020 & Martín Emilio Beltrán \\
\hline 2019 & $\begin{array}{l}\text { SL1679- } \\
2019\end{array}$ & Clara Cecilia Dueñas & 2020 & SL570-2020 & Martín Emilio Beltrán \\
\hline 2019 & $\begin{array}{c}\text { SL1826- } \\
2019\end{array}$ & Ana María Muñoz Segura & 2020 & SL560-2020 & Jimena Isabel Godoy \\
\hline 2019 & $\begin{array}{l}\text { SL1267- } \\
2019\end{array}$ & Jimena Isabel Godoy & 2020 & SL599-2020 & Ana María Muñoz Segura \\
\hline
\end{tabular}

Via Inveniendi Et Iudicandi

e-ISSN: 1909-0528 | DOI: https://doi.org/10.15332/19090528

Vol. 16 N. ${ }^{\circ} 2$ | julio-diciembre del 2021 


\begin{tabular}{|c|c|c|c|c|c|}
\hline Año & $\begin{array}{c}\text { N. }{ }^{\circ} \text { de } \\
\text { sentencia }\end{array}$ & Magistrado ponente & Año & $\begin{array}{c}\text { N. }{ }^{\circ} \text { de } \\
\text { sentencia }\end{array}$ & Magistrado ponente \\
\hline 2019 & $\begin{array}{l}\text { SL1037- } \\
2019\end{array}$ & Jimena Isabel Godoy & 2020 & SL450-2020 & Cecilia Margarita Durán \\
\hline 2019 & $\begin{array}{l}\text { SL1047- } \\
2019\end{array}$ & Donald José Dix Ponnefz & 2020 & SL354-2020 & Martín Emilio Beltrán \\
\hline 2019 & SL904-2019 & Cecilia Margarita Durán & 2020 & SL326-2020 & Jimena Isabel Godoy \\
\hline 2019 & SL902-2019 & Cecilia Margarita Durán & 2020 & SL249-2020 & Jorge Prada Sánchez \\
\hline 2019 & $\begin{array}{l}\text { SL1361- } \\
2019\end{array}$ & Gerardo Botero Zuluaga & 2020 & SL178-2020 & Omar de Jesús Restrepo \\
\hline
\end{tabular}

Fuente: Corte Suprema de Justicia (s. f.).

Falta de entrega de elementos de protección personal

"No le proporcionó protectores o mascarillas que evitaran la lesión" (C.S.J., Sentencia SL5252-2019, Colom., pp. 2-3); "no suministró los elementos de protección adecuados” (C.S.J., SL4455-2019, Colom., p. 2); "la empresa lo contrató sin equipo para realizar la vigilancia del lugar" (C.S.J., SL4473-2019, Colom.); "uso de elemento de protección inadecuado para la manipulación de la lámina” (C.S.J., SL4965-2019, Colom., p. 3); “el causante ingresó a un silo por su parte superior sin la debida máscara protectora y sin cinturón de seguridad" (C.S.J., SL3664-2019, Colom., p. 3); "a los trabajadores no les fueron suministrados los elementos de seguridad necesarios para realizar la labor" (C.S.J., SL3672-2019, Colom., p. 3); y "el empleador lo obligó a realizar su labor en un sitio de alto riesgo, desprovisto de elementos de seguridad que impidieran que cayera y muriera" (C.S.J., SL3897-2019, Colom., p. 2).

Falta de capacitación

"La empresa lo contrató sin capacitaciones" (C.S.J., SL4473-2019, Colom.); “Agarre de lámina en forma errada por falta de conocimiento al manipular este material” (C.S.J., 4965-2019, Colom., p. 3); “No le capacitó suficientemente" (C.S.J., SL3897-2019, Colom., p. 2); "Falta de capacitación del trabajador” (C.S.J., SL3214-2019, Colom.); “No haber recibido el trabajador por parte del empleador al inicio de la contratación 
capacitación alguna" (C.S.J., SL3498-2019, Colom., p. 6) y "no fue capacitado para que desarrollara a cabalidad esta labor" (C.S.J., SL26802019, Colom., p. 3).

\section{Falta de controles administrativos de seguridad}

"No se previó un cruce o acercamiento de una línea de electricidad" (C.S.J., SL2783-2019, Colom.); "no elaboró estudios de suelo, previo a la ejecución de las obras" (C.S.J., 4455-2019, Colom., p. 2); "sin la mínima precaución del empleador, se envió a un sitio de la vía pública con mucho flujo vehicular" (C.S.J., 3915-2019, Colom., p. 2) y "no había señalización en la vía, los trabajadores solo tenían chaleco, no tenían bombones, ese día solo había avisos en la carretera" (C.S.J., SL1999-2019, Colom., pp. 6-7).

Falta de controles de riesgos químicos y físicos

"La mina no contaba con un buen sistema de ventilación, que uno de los ventiladores estaba malo, que las mediciones de gas no se hacían de manera que garantizaban la seguridad de los mineros, que no había [sic] medición de polvo de carbón y polvo de roca" (C.S.J., SL3693-2019, Colom., pp. 2-3).

Materiales peligrosos

"Material flexible y cortante" (C.S.J., SL4965-2019, Colom., p. 3) y "explosión de dinamita en el sitio de trabajo" (C.S.J., SL2206-2019, Colom., p. 2).

Falta de procedimientos e instructivos de seguridad y salud en el trabajo

"La empresa de Carbones no tenía protocolos para medir gas metano más allá de la altura con la mano" (C.S.J., SL3693-2019, Colom., p. 2). "la operación se estaba efectuando con algunos inconvenientes de seguridad, además, no se tomaron medidas de seguridad, ni se atendieron los

Via Inveniendi Et Iudicandi

e-ISSN: 1909-0528 | DOI: https://doi.org/10.15332/19090528

Vol. 16 N.o 2 | julio-diciembre del 2021 
reclamos de los trabajadores ante la peligrosidad” (C.S.J., SL2680-2019, Colom., pp. 4-5); "no se contaba con un manual de procedimientos para las solicitudes de libranzas entre las subestaciones" (C.S.J., SL2783-2019, Colom., p. 3); "el automotor no tenía sistema de alarma o sonido que alertara a quienes se encontraban cerca" (C.S.J., SL1911-2019, Colom., p. 4); y "falta de procedimiento escrito y claro para la realización de este tipo de labores" (C.S.J., SL3588-2019, Colom., p. 8).

Instalaciones, maquinarias y equipos en mal estado

"Cables desentubados, bornera de empalme partida en la parte interior y exterior, tablero de mando con presencia de corrosión y sin identificación de cuñas" (C.S.J., SL3214-2019, Colom., p. 3); "se reventó el cinturón de seguridad que lo sujetaba, por el mal estado en que se encontraba" (C.S.J., SL2426-2019, Colom., p. 2); "la pluma no aguantó el peso que manejaba y no se tomaron medidas para resguardar el punto de operación de la máquina, lo que generó un peligro inminente para el operario" (C.S.J., SL1679-2019, Colom., p. 4); "omisión de la empleadora en el deber de diligencia y cuidado del mantenimiento de los equipos para garantizar su debido funcionamiento" (C.S.J., SL1826-2019, Colom., p. 2).

Por tanto, nos muestra que las causas son la falta de elementos de protección personal, de controles administrativos de seguridad y la falta de procedimientos e instructivos de seguridad y salud en el trabajo todas relacionadas a la falta de gestión de peligros y riesgos y a la clasificación de peligros y riesgos de condiciones de seguridad.

\section{Categorías de las Consideraciones / razón de la decisión}

Falta dotación y uso de elementos de protección personal

"Falta de supervisión y uso de los elementos de protección personal (gafas)" (C.S.J., SL5252-2019, Colom., p. 12); "no tenía ni arnés, ni cinturón de seguridad adecuado" (C.S.J., SL4653-2019, Colom., p. 8); “no

Via Inveniendi Et Iudicandi

e-ISSN: 1909-0528 | DOI: https://doi.org/10.15332/19090528

Vol. 16 N.o 2 | julio-diciembre del 2021 
había línea de vida" (C.S.J., SL4653-2019, Colom., p. 8); "la accionada incumplió las normas de seguridad dotando de un elemento inseguro y que no cumplía las descripciones, tampoco existía la línea de vida" (C.S.J., SL4653-2019, Colom., p. 9); "se le obligó al trabajador a permanecer allí, sin supervisión, sin ninguna protección y sin la fijación de estándares mínimos de seguridad" (C.S.J., SL4455-2019, Colom., p. 5); "se omitió brindarle los elementos de protección personal” (C.S.J., SL4965-2019, Colom.) y "no demostró haberle suministrado a su trabajador la capacitación necesaria, ni la dotación” (C.S.J., SL3897-2019, Colom., p. 10); "el protocolo en alta mar, por lo menos exige que se dote al trabajador de un chaleco salvavidas, y en este caso no se demostró haberlo entregado" (C.S.J., SL3897-2019, Colom., p. 10).

Falta de supervisión por parte del empleador

"Falta de supervisión y uno de los elementos de protección personal (gafas) al momento de ejercer el ex trabajador la labor encomendada" (C.S.J., SL5252-2019, Colom., p. 12); "no hubo la vigilancia continua mientras realizaba la tarea" (C.S.J., SL4653-2019, Colom., p. 8) y “a pesar de los riesgos del lugar de trabajo, se le obligó al trabajador a permanecer allí sin ninguna protección y sin la fijación de estándares mínimos de seguridad" (C.S.J., SL4455-2019, Colom., p. 5); y "no haber adoptado las medidas específicas para prevenir el peligro, como la de hacerlo acompañar de supervisión" (C.S.J., SL4455-2019, Colom., pp. 5-6).

Falta de identificación de peligros y riesgos

"El empleador omitió establecer, un panorama general de los factores de riesgo, y el grado y clasificación del peligro" (C.S.J., SL4811-2019, Colom., pp. 17-18) y "la causa determinante de ese accidente, fue la conducta patronal negligente, al no haber efectuado una estimación razonable del riesgo al que estaba sometido el trabajador" (C.S.J., SL4455-2019, p. 5). 
Falta de implementación de medidas preventivas frente a peligros y riesgos identificados

"Adopción de las medidas de protección y control para minimizar los daños y consecuencias” (C.S.J., SL4811-2019, Colom., p. 18); “omitir la colocación de avisos, señales, tarjetas, etc.” (C.S.J., SL3915-2019, Colom., p. 7); "Debieron colocarse conos de prevención en los extremos del vehículo como señal a los conductores que transitaban por la vía” (C.S.J., SL3915-2019, Colom., p. 9); y "estas circunstancias eran controlables o previsibles por la empresa, sin embargo, omitió obrar con la diligencia que le correspondía” (C.S.J., SL3664-2019, Colom., p. 12).

Falta de mantenimiento e inspección de instalaciones, maquinarias y equipos

"Según las fotografías traídas al plenario, no reflejaban un sitio óptimo de trabajo" (C.S.J., SL4653-2019, Colom., p. 8).

Falta de procedimientos e instructivos internos de seguridad y salud en el trabajo

"Omitió una de las reglas de oro de la seguridad eléctrica" (C.S.J., SL48492019, Colom., p. 22); "se debió establecer un procedimiento que permita maniobrar la palanca desde otro punto" (C.S.J., SL3915-2019, Colom., p. 8) y "debía contar con un procedimiento y un protocolo de seguridad claro y preciso" (C.S.J., SL3588-2019, Colom., p. 8).

Falta de capacitación del trabajador

"Se contrató a una persona para realizar la vigilancia del lugar, sin tener en cuenta que estas personas deben tener una capacitación especial" (C.S.J., SL4473-2019,Colom.); "se omitió capacitar al aprendiz" (C.S.J., SL49652019, Colom.) y "no demostró haberle suministrado a su trabajador la capacitación necesaria” (C.S.J., SL3897-2019, Colom., p. 10) y “el deber

Via Inveniendi Et Iudicandi

e-ISSN: 1909-0528 | DOI: https://doi.org/10.15332/19090528

Vol. 16 N.० 2 | julio-diciembre del 2021 
del empleador de capacitar a su trabajador no se contrae a las funciones de la labor contratada, sino también el uso de herramientas y máquinas de trabajo frente a los riesgos ocupacionales a los que se encuentra expuesto" (C.S.J., SL3536-2019, Colom., p. 21).

Falta de controles de ingeniería

"Equívoca apreciación de los controles de seguridad sobre nivel de medición de gases” (C.S.J., SL3693-2019, Colom., p. 23). En la codificación anterior nos muestra que las categorías más relevantes son la falta de elementos de protección personal, la falta de implementación de medidas de prevención y control frente a peligros y riesgos identificados. 


\section{Capítulo 2. Interpretación global-aproximación explicativa}

Figura 1. Diagrama de interpretación global/sentencias por culpa patronal por accidente de trabajo

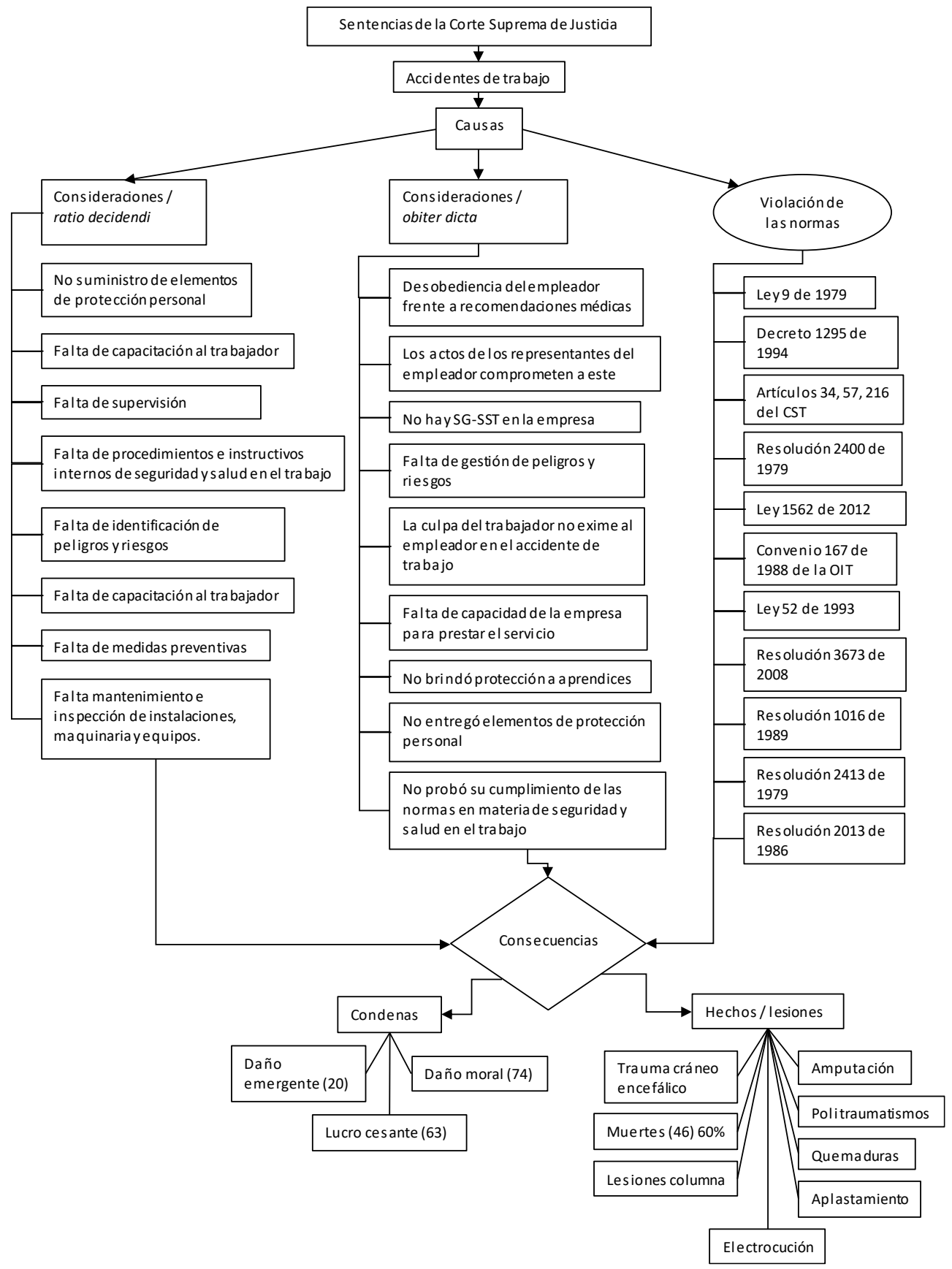

Fuente: elaboración propia.

Via Inveniendi Et Iudicandi

e-ISSN: 1909-0528 | DOI: https://doi.org/10.15332/19090528

Vol. 16 N.0 2 | julio-diciembre del 2021 
En la figura 1 se hizo una aproximación a la interpretación global de todos los resultados, haciendo una interrelación de los hechos, consideraciones, los resuelve y normas jurídicas en las que, finalmente, argumentan la decisión de la culpa patronal por accidente de trabajo.

Por otro lado, en las consideraciones de dichas sentencias, en la razón de la decisión incluyen los hallazgos concernientes a varias de las obligaciones de un Sistema de Gestión de Seguridad y Salud en el Trabajo en la fase del hacer correspondientes a la gestión de los peligros y los riesgos laborales como el no suministro de elementos de protección personal, falta de diligencia y cuidado, conducta omisiva del empleador, falta de supervisión de normas, falta de protocolos de seguridad, falta de capacitación y entrenamiento, falta de herramientas y equipos seguros. También, obligaciones en relación con la fase del planear como falta de programas de seguridad y salud en el trabajo y faltan programas de seguridad. Igualmente, hicieron énfasis en aspectos como la conducta negligente y descuidada; lo cual nos muestra que la mayoría de estos accidentes de trabajo, severos y mortales, ocurren debido a que el empleador no cumple con sus obligaciones en materia de seguridad y salud en el trabajo. Es decir, no hubo un enfoque preventivo desde el control y la gestión de los peligros y los riegos laborales. Todo esto fue argumentado y fundamentado a través de algunas normas anteriores a la Resolución 0312 de 2019 (estándares mínimos del Sistema de Gestión de Seguridad y Salud en el Trabajo), pero que estas normas preliminares se encuentran incluidas en dicha resolución y todas están vigentes, como la Ley 9 de 1979, Resolución 1016 de 1989, Decreto 1295 de 1994, Resolución 2400 de 1989, Ley 1562 de 2012 y la Resolución 2013 de 1986, entre otras.

En la figura 2 se mostró que, al comparar las consideraciones de las sentencias y el porcentaje de observancia de los jueces sobre el cumplimiento por parte de los empleadores, con referencia a las

Via Inveniendi Et Iudicandi

e-ISSN: 1909-0528 | DOI: https://doi.org/10.15332/19090528

Vol. 16 N.o 2 | julio-diciembre del 2021 
obligaciones en riesgos laborales de la normatividad anterior mencionada con los estándares mínimos específicos en gestión de peligros y riesgos del Sistema de Gestión de Seguridad y Salud en el Trabajo (SG-SST) (Resolución 0312 de 2019), se encontró similitud, correlación y concordancia.

Figura 2. Diagrama de aproximación explicativa. Sentencias / culpa patronal / observancias-concordancia con estándares mínimos del Sistema de Gestión de Seguridad y Salud en el Trabajo

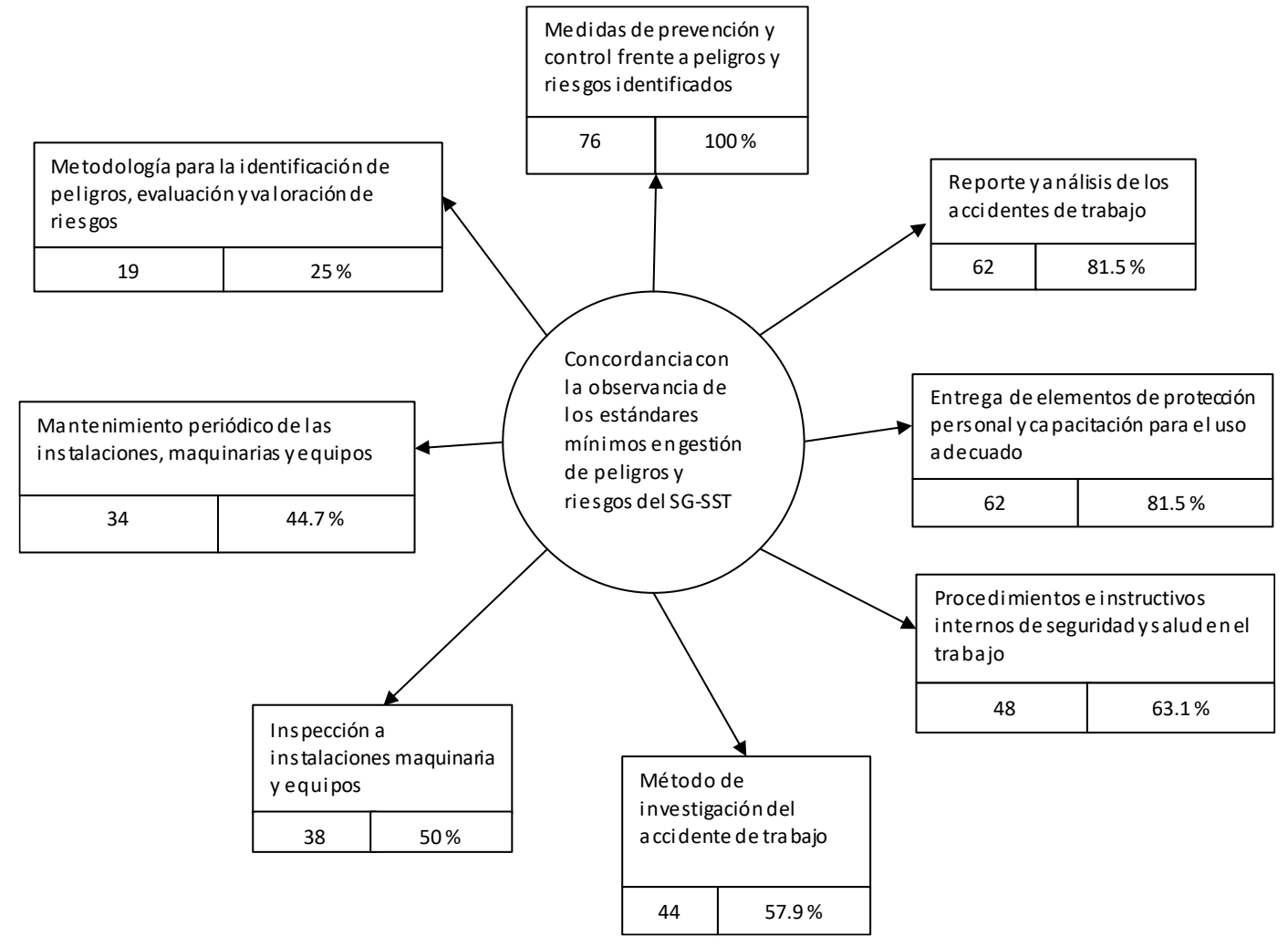

Fuente: elaboración propia.

Se encontró que la mayor observancia y requerimientos solicitados a los empleadores fueron las medidas de prevención y control frente a los peligros y los riesgos identificados, seguido por reportes y análisis de los accidentes de trabajo, entrega de elementos de protección personal y la capacitación para el uso adecuado, procedimientos e instructivos internos

Via Inveniendi Et Iudicandi

e-ISSN: 1909-0528 | DOI: https://doi.org/10.15332/19090528

Vol. 16 N.o 2 | julio-diciembre del 2021 
en seguridad y salud en el trabajo, métodos de investigación de los accidentes de trabajo, inspección a instalaciones, maquinarias y equipos como el mantenimiento periódico de instalaciones, maquinarias y equipos. Es así como encontramos que corresponden a los estándares mínimos fase del Hacer específicamente, donde están los requerimientos a los empleadores del desarrollo y ejecución de los programas orientados a la gestión de peligros y riesgos orientados a la prevención y protección de la población trabajadora, aspectos cruciales coherentes con la alta peligrosidad de dichas empresas en las clases de riesgo $\mathrm{IV}$ y $\mathrm{V}$, condenadas por culpa patronal.

\section{Capítulo 3. Discusión}

Las normas de seguridad y salud en el trabajo que fueron revisadas en las sentencias fueron: el literal c del artículo 21 del Decreto 1295 de 1994, el literal b del artículo 2 de la Resolución 2400 de 1979, los literales a y d del artículo 84 de la Ley 9 de 1979, el artículo 40 de Resolución 2413 de 1979, el artículo 11, numerales 2, 5, 7, 9, 11 de la Resolución 1016 de 1989, relacionados con la instalación y mantenimiento de instalaciones maquinarias y equipos; el literal g del artículo 21 del Decreto 1295 de 1994, el literal g del artículo 2, y los artículos 188 y 90 de la Resolución 2400 de 1979, el numeral 6 de la Circular unificada del 22 de abril de 2004 de la Dirección General de Riesgos Profesionales, el artículo 84, literal g de la Ley 9 de 1979, el artículo 13 de la Resolución 3673 de 2008, el artículo 11, numerales 12 y 13 de la Resolución 1016 de 1989, relacionados con la entrega de elementos de protección personal y capacitación para su uso adecuado; el artículo 22, literal d del Decreto 1295 de 1994, relacionado con la adopción de las medidas de prevención y control por parte de los trabajadores; el artículo 56 del Decreto 1295 de 1994, el artículo 2 de la Ley 52 de 1993, el artículo 11, numerales 6, 10, 17 y 18 de la Resolución 1016 de 
1989, relacionados con las medidas de prevención y control frente a peligros y riesgos identificados; el artículo 2, literal f de la Resolución 2400 de 1979, el artículo 84 literal a de la Ley 9 de 1979, el artículo 15 de la Resolución 180398 del 7 de abril de 2004, el artículo 11, numeral 8 de la Resolución 1016 de 1989, relacionados con procedimientos e instructivos de seguridad y salud en el trabajo; el 84 literal e de la Ley 9 de 1979, el artículo 11, numerales 14 y 15 de la Resolución 1016 de 1989, relacionados con reporte, investigación y análisis de los accidentes de trabajo; y el artículo 11, numerales 1, 3 y 4 de la Resolución 1016 de 1989, todas relacionadas con identificación de peligros y riesgos, normas precedentes, pero vigentes, se encontró que están contenidas en los estándares mínimos de los sistemas de gestión de la seguridad y salud en el trabajo de la Resolución 0312 de 2019.

Se compararon los resultados desde el enfoque teórico y se encontró que eran coherentes con la teoría de culpa patronal desde el riesgo creado, pues tal como lo explica Yong (2019) y Maldonado (2017), el empleador es responsable si no ha verificado que las condiciones en la que está el trabajador son adecuadas para su salud y seguridad. Esta implica que, tal como lo explica Sánchez (2015), el juez debe valorar, de manera objetiva, el cumplimiento de las obligaciones en riesgos laborales, en especial lo concerniente a la gestión y prevención de los peligros y riesgos laborales, los cuales están implícitos en los estándares mínimos del Sistema de Gestión de Seguridad y Salud en el Trabajo, y esto es lo que ha hecho la Corte Suprema de Justicia, entendiendo que cuando el empleador incumple con sus obligaciones, en materia de seguridad y salud en el trabajo, está creando un riesgo que se materializa en la culpa patronal (Feria, 2020).

Esta investigación mostró que la Corte Suprema de Justicia, sin necesidad de caer en la responsabilidad objetiva, estudia la culpa patronal desde un 
análisis objetivo del cumplimiento de la gestión y prevención de los peligros y riesgos laborales, los cuales forman parte fundamental de los estándares mínimos del Sistema de Gestión de Seguridad y Salud en el Trabajo, aplicando así la teoría del riesgo creado, adoptada por este trabajo.

Finalmente, esta investigación encontró consistencias, como lo mencionado por Sánchez (2015), en la que la culpa patronal debe analizarse desde una evaluación objetiva del cumplimiento de las obligaciones del empleador en materia de seguridad y salud en el trabajo, esto sin querer llevar el estudio de la culpa patronal a un marco de responsabilidad objetiva. Lo anterior con el fin de evitar graves accidentes como los analizados en este estudio, en los que un 60.5\% de las lesiones acabaron con la vida de los trabajadores.

\section{Conclusión}

Aunque no se mencionó la Resolución 0312 de 2019 por el incumplimiento de los estándares mínimos de seguridad y salud en el trabajo, sí se argumentó que hubo conducta negligente y descuidada, lo cual nos muestra que la mayoría de estos accidentes de trabajo severos y mortales ocurrieron debido a que el empleador no tuvo en cuenta un enfoque preventivo desde el control y la gestión de peligros y riesgos laborales, que es congruente con los estándares mínimos del Sistema de Gestión de Seguridad y Salud en el Trabajo mencionados. Además, en amplios porcentajes, como es el caso de las medidas de prevención y control frente a peligros y riesgos identificados, reporte y análisis de los accidentes de trabajo y entrega de elementos de protección personal y capacitación para el uso adecuado.

De igual manera, es necesario resaltar que se encontró un déficit jurisprudencial, ya que a pesar de que algunas sentencias analizadas

Via Inveniendi Et Iudicandi

e-ISSN: 1909-0528 | DOI: https://doi.org/10.15332/19090528

Vol. 16 N.० 2 | julio-diciembre del 2021 
resuelven conflictos relativos a accidentes de trabajo ocurridos con posterioridad al 2012, no se menciona la Ley 1562 de 2012 en la parte argumentativa de dichas sentencias.

\section{Referencias}

Colombia. Ley 9 de 1979. Diario Oficial n. ${ }^{\circ}$ 35308. Julio 16 de 1979. http://www.secretariasenado.gov.co/senado/basedoc/ley_ooog_1979.html

Colombia. Ley 1562 de 2012. Diario Oficial n. ${ }^{\circ}$ 48.488. Julio 11 de 2012. http://secretariasenado.gov.co/senado/basedoc/ley 1562 2012.html

Colombia. Ley 52 de 1993. Diario Oficial n. ${ }^{\circ}$ 40.914. Junio 9 de 1993. https://www.minsalud.gov.co/sites/rid/Lists/BibliotecaDigital/RIDE/DE/DIJ/Le y-0052-DE-1993.pdf

Colombia. Decreto Ley 3743 de 1950. Código Sustantivo del Trabajo [C.S.T.]. Diario Oficial n. ${ }^{\circ}$ 27.622. Junio 7 de 1951. http://www.secretariasenado.gov.co/senado/basedoc/codigo sustantivo trabajo. $\underline{\mathrm{html} \# 1}$

Corte Suprema de Justicia [C.S.J.], Sala Laboral, diciembre 2, 2019, M.P.: C. A. Guarín Jurado, Sentencia SL5252-2019, [Colom.].

Corte Suprema de Justicia [C.S.J.], Sala Laboral, noviembre 6, 2019, M.P.: E. Forero Vargas, Sentencia SL4811-2019, [Colom.].

Corte Suprema de Justicia [C.S.J.], Sala Laboral, octubre 29, 2019, M.P.: E. Forero Vargas, Sentencia SL4653-2019, [Colom.].

Corte Suprema de Justicia [C.S.J.], Sala Laboral, octubre 22, 2019, M.P.: G. F. Rodríguez Jiménez, Sentencia SL4849-2019, [Colom.].

Corte Suprema de Justicia [C.S.J.], Sala Laboral, octubre 16, 2019, M.P.: J. Prada Sánchez, Sentencia SL4455-2019, [Colom.].

Corte Suprema de Justicia [C.S.J.], Sala Laboral, octubre 16, 2019, M.P.: O. De J. Restrepo Ochoa, Sentencia SL4473-2019, [Colom.].

Corte Suprema de Justicia [C.S.J.], Sala Laboral, octubre 8, 2019, M.P.: A. M. Muñoz Segura, Sentencia SL4965-2019, [Colom.].

Via Inveniendi Et Iudicandi e-ISSN: 1909-0528 | DOI: https://doi.org/10.15332/19090528 Vol. 16 N.० 2 | julio-diciembre del 2021 
Corte Suprema de Justicia [C.S.J.], Sala Laboral, septiembre 18, 2019, M.P.: C. C. Dueñas Quevedo, Sentencia SL4570-2019, [Colom.].

Corte Suprema de Justicia [C.S.J.], Sala Laboral, septiembre 10, 2019, M.P.: C. M. Durán Ujueta, Sentencia SL3897-2019, [Colom.].

Corte Suprema de Justicia [C.S.J.], Sala Laboral, septiembre 3, 2019, M.P.: S. R. Brito Cuadrado, Sentencia SL3915-2019, [Colom.].

Corte Suprema de Justicia [C.S.J.], Sala Laboral, septiembre 3, 2019, M.P.: D. A. Caguasango Villota, Sentencia SL3664-2019, [Colom.].

Corte Suprema de Justicia [C.S.J.], Sala Laboral, agosto 28, 2019, M.P.: A. M. Muñoz Segura, Sentencia SL3693-2019, [Colom.].

Corte Suprema de Justicia [C.S.J.], Sala Laboral, agosto 28, 2019, M.P.: D. J. Dix Ponnefz, Sentencia SL3536-2019, [Colom.].

Corte Suprema de Justicia [C.S.J.], Sala Laboral, agosto 28, 2019, M.P.: G. F. Rodríguez Jiménez, Sentencia SL3588-2019, [Colom.].

Corte Suprema de Justicia [C.S.J.], Sala Laboral, agosto 28, 2019, M.P.: J. I. Godoy Fajardo, Sentencia SL3672-2019, [Colom.].

Corte Suprema de Justicia [C.S.J.], Sala Laboral, agosto 28, 2019, M.P.: C. A. Guarín Jurado, Sentencia SL3537-2019, [Colom.].

Corte Suprema de Justicia [C.S.J.], Sala Laboral, agosto 6, 2019, M.P.: Dolly Amparo Caguasango Villota, Sentencia SL3214-2019, [Colom.].

Corte Suprema de Justicia [C.S.J.], Sala Laboral, agosto 6, 2019, M.P.: E. F. Vargas, Sentencia SL3498-2019, [Colom.].

Corte Suprema de Justicia [C.S.J.], Sala Laboral, julio 4, 2019, M.P.: J. I. Godoy Fajardo, Sentencia SL2777-2019, [Colom.].

Corte Suprema de Justicia [C.S.J.], Sala Laboral, julio 24, 2019, M.P.: J. I. Godoy Fajardo, Sentencia SL2782-2019, [Colom.].

Corte Suprema de Justicia [C.S.J.], Sala Laboral, julio 17, 2019, M.P.: D. J. Dix Ponnefz, Sentencia SL2831-2019, [Colom.].

Corte Suprema de Justicia [C.S.J.], Sala Laboral, julio 17, 2019, M.P.: J. I. Godoy Fajardo, Sentencia SL2680-2019, [Colom.].

Via Inveniendi Et Iudicandi

e-ISSN: 1909-0528 | DOI: https://doi.org/10.15332/19090528

Vol. 16 N.o 2 | julio-diciembre del 2021 
Corte Suprema de Justicia [C.S.J.], Sala Laboral, M.P.julio 16, 2019,: C. M. Durán Ujueta, Sentencia SL2689-2019, [Colom.].

Corte Suprema de Justicia [C.S.J.], Sala Laboral, julio 9, 2019, M.P.: O. De J. Restrepo Ochoa, Sentencia SL2783-2019, [Colom.].

Corte Suprema de Justicia [C.S.J.], Sala Laboral, julio 3, 2019, M.P.: J. P. Sánchez, Sentencia SL2426-2019, [Colom.].

Corte Suprema de Justicia [C.S.J.], Sala Laboral, mayo 29, 2019, M.P.: M. E. Beltrán Quintero, Sentencia SL1848-2019, [Colom.].

Corte Suprema de Justicia [C.S.J.], Sala Laboral, mayo 29, 2019, M.P.: D. J. Dix Ponnefz, Sentencia SL1999-2019, [Colom.].

Corte Suprema de Justicia [C.S.J.], Sala Laboral, mayo 22, 2019, M.P.: C. C. Dueñas Quevedo, Sentencia SL1911-2019, [Colom.].

Corte Suprema de Justicia [C.S.J.], Sala Laboral, mayo 22, 2019, M.P.: J. L. Quiroz Alemán, Sentencia SL2206-2019, [Colom.].

Corte Suprema de Justicia [C.S.J.], Sala Laboral, abril 30, 2019, M.P.: J. I. Godoy Fajardo, Sentencia SL1581-2019, [Colom.].

Corte Suprema de Justicia [C.S.J.], Sala Laboral, abril 30, 2019, M.P.: C. C. Dueñas Quevedo, Sentencia SL1679-2019, [Colom.].

Corte Suprema de Justicia [C.S.J.], Sala Laboral, abril 23, 2019, M.P.: A. M. Muñoz Segura, Sentencia SL1826-2019, [Colom.].

Corte Suprema de Justicia [C.S.J.], Sala Laboral, abril 10, 2019, M.P.: J. I. Godoy Fajardo, Sentencia SL1267-2019, [Colom.].

Corte Suprema de Justicia [C.S.J.], Sala Laboral, marzo 27, 2019, M.P.: J. I. Godoy Fajardo, Sentencia SL1037-2019, [Colom.].

Corte Suprema de Justicia [C.S.J.], Sala Laboral, marzo 27, 2019, M.P.: D. J. Dix Ponnefz, Sentencia SL1047-2019, [Colom.].

Corte Suprema de Justicia [C.S.J.], Sala Laboral, marzo 19, 2019, M.P.: C. M. Durán Ujueta, Sentencia SL904-2019, [Colom.].

Corte Suprema de Justicia [C.S.J.], Sala Laboral, marzo 19, 2019, M.P.: C. M. Durán Ujueta, Sentencia SL902-2019, [Colom.].

Via Inveniendi Et Iudicandi

e-ISSN: 1909-0528 | DOI: https://doi.org/10.15332/19090528

Vol. 16 N.o 2 | julio-diciembre del 2021 
Corte Suprema de Justicia [C.S.J.], Sala Laboral, marzo 13, 2019, M.P.: G. Botero Zuluaga, Sentencia SL1361-2019, [Colom.].

Corte Suprema de Justicia [C.S.J.], Sala Laboral, marzo 13, 2019, M.P.: J. I. Godoy Fajardo, Sentencia SL786-2019, [Colom.].

Corte Suprema de Justicia [C.S.J.], Sala Laboral, marzo 5, 2019, M.P.: O. De J. Restrepo Ochoa, Sentencia SL795-2019, [Colom.].

Corte Suprema de Justicia [C.S.J.], Sala Laboral, marzo 5, 2019, M.P.: C. M. Durán Ujueta, Sentencia SL651-2019, [Colom.].

Corte Suprema de Justicia [C.S.J.], Sala Laboral, febrero 27, 2019, M.P.: J. Prada Sánchez, Sentencia SL583-2019, [Colom.].

Corte Suprema de Justicia [C.S.J.], Sala Laboral, febrero 27, 2019, M.P.: J. I. Godoy Fajardo, Sentencia SL566-2019, [Colom.].

Corte Suprema de Justicia [C.S.J.], Sala Laboral, febrero 20, 2019, M.P.: E. Forero Vargas, Sentencia SL445-2019, [Colom.].

Corte Suprema de Justicia [C.S.J.], Sala Laboral, febrero 13, 2019, M.P.: E. Forero Vargas, Sentencia SL354-2019, [Colom.].

Corte Suprema de Justicia [C.S.J.], Sala Laboral, agosto 19, 2020, M.P.: D. A. Caguasango Villota, Sentencia SL3156-2020, [Colom.].

Corte Suprema de Justicia [C.S.J.], Sala Laboral, agosto 19, 2020, M.P.: D. A. Caguasango Villota, Sentencia SL3048-2020, [Colom.].

Corte Suprema de Justicia [C.S.J.], Sala Laboral, agosto 11, 2020, M.P.: O. De J. Restrepo Ochoa, Sentencia SL2906-2020, [Colom.].

Corte Suprema de Justicia [C.S.J.], Sala Laboral, julio 28, 2020, M.P.: O. De J. Restrepo Ochoa, Sentencia SL2653-2020, [Colom.].

Corte Suprema de Justicia [C.S.J.], Sala Laboral, julio 13, 2020, M.P.: S. R. Brito Cuadrado, Sentencia SL2629-2020, [Colom.].

Corte Suprema de Justicia [C.S.J.], Sala Laboral, junio 24, 2020, M.P.: A. M. Muñoz Segura, Sentencia SL2388-2020, [Colom.].

Corte Suprema de Justicia [C.S.J.], Sala Laboral, junio 24, 2020, M.P.: F. Castillo Cadena, Sentencia SL2335-2020, [Colom.].

Via Inveniendi Et Iudicandi

e-ISSN: 1909-0528 | DOI: https://doi.org/10.15332/19090528

Vol. 16 N.0 2 | julio-diciembre del 2021 
Corte Suprema de Justicia [C.S.J.], Sala Laboral, junio 16, 2020, M.P.: S. R. Brito Cuadrado, Sentencia SL2491-2020, [Colom.].

Corte Suprema de Justicia [C.S.J.], Sala Laboral, mayo 27, 2020, M.P.: O. de J. Restrepo Ochoa, Sentencia SL1906-2020, [Colom.].

Corte Suprema de Justicia [C.S.J.], Sala Laboral, mayo 27, 2020, M.P.: M. E. Beltrán Quintero, Sentencia SL1565-2020, [Colom.].

Corte Suprema de Justicia [C.S.J.], Sala Laboral, mayo 20, 2020, M.P.: J. I. Godoy Fajardo, Sentencia SL1598-2020, [Colom.].

Corte Suprema de Justicia [C.S.J.], Sala Laboral, mayo 19, 2020, M.P.: A. M. Muñoz Segura, Sentencia SL1743-2020, [Colom.].

Corte Suprema de Justicia [C.S.J.], Sala Laboral, mayo 19, 2020, M.P.: M. E. Beltrán Quintero, Sentencia SL1558-2020, [Colom.].

Corte Suprema de Justicia [C.S.J.], Sala Laboral, mayo 13, 2020, M.P.: D. J. Dix Ponnefz, Sentencia SL1608-202O, [Colom.].

Corte Suprema de Justicia [C.S.J.], Sala Laboral, mayo 5, 2020, M.P.: D. A. Caguasango Villota, Sentencia SL1388-202O, [Colom.].

Corte Suprema de Justicia [C.S.J.], Sala Laboral, abril 29, 2020, M.P.: J. Prada Sánchez, Sentencia SL1438-202O, [Colom.].

Corte Suprema de Justicia [C.S.J.], Sala Laboral, abril 22, 2020, M.P.: J. Prada Sánchez, Sentencia SL1426-202O, [Colom.].

Corte Suprema de Justicia [C.S.J.], Sala Laboral, abril 21, 2020, M.P.: C. A. Guarín Jurado, Sentencia SL1465-202O, [Colom.].

Corte Suprema de Justicia [C.S.J.], Sala Laboral, abril 20, 2020, M. P.: M. E. Beltrán Quintero, Sentencia SL1336-2020, [Colom.].

Corte Suprema de Justicia [C.S.J.], Sala Laboral, Magistrado Ponente: Martín Emilio Beltrán Quintero, abril 20, 2020, Sentencia SL1O83-202O, [Colom.].

Corte Suprema de Justicia [C.S.J.], Sala Laboral, marzo 10, 2020, M.P.: A. M. Muñoz Segura, Sentencia SL1187-2O2O, [Colom.].

Corte Suprema de Justicia [C.S.J.], Sala Laboral, febrero 5, 2020, M.P.: J. Prada Sánchez, Sentencia SL249-202O, [Colom.].

Via Inveniendi Et Iudicandi

e-ISSN: 1909-0528 | DOI: https://doi.org/10.15332/19090528

Vol. 16 N.० 2 | julio-diciembre del 2021 
Corte Suprema de Justicia [C.S.J.], Sala Laboral, marzo 4, 2020, M.P.: Jimena Isabel Godoy Fajardo, Sentencia SL696-202O, [Colom.].

Corte Suprema de Justicia [C.S.J.], Sala Laboral, febrero 26, 2020, M.P.: M. E. Beltrán Quintero, Sentencia SL573-2020, [Colom.].

Corte Suprema de Justicia [C.S.J.], Sala Laboral, febrero 26, 2020, M.P.: M. E. Beltrán Quintero, Sentencia SL57O-2020, [Colom.].

Corte Suprema de Justicia [C.S.J.], Sala Laboral, febrero 26, 2020, M.P.: J. I. Godoy Fajardo, Sentencia SL560-2020, [Colom.].

Corte Suprema de Justicia [C.S.J.], Sala Laboral, febrero 18, 2020, M.P.: A. M. Muñoz Segura, Sentencia SL599-2020, [Colom.].

Corte Suprema de Justicia [C.S.J.], Sala Laboral, febrero 17, 2020, M.P.: C. M. Durán Ujueta, Sentencia SL45O-2O2O, [Colom.].

Corte Suprema de Justicia [C.S.J.], Sala Laboral, febrero 12, 2020, M.P.: M. E. Beltrán Quintero, Sentencia SL354-202O, [Colom.].

Corte Suprema de Justicia [C.S.J.], Sala Laboral, febrero 12, 2020, M.P.: J. I. Godoy Fajardo, Sentencia SL326-2O2O, [Colom.].

Corte Suprema de Justicia [C.S.J.], Sala Laboral, enero 28, 2020, M.P.: O. De J. Restrepo Ochoa, Sentencia SL178-2O2O, [Colom.].

Decreto 1072 de 2015 [Ministerio del Trabajo]. Por medio del cual se expide el Decreto Único Reglamentario del Sector Trabajo. 26 de mayo de 2015. https://www.mintrabajo.gov.co/documents/20147/o/DUR+Sector+Trabajo+Actu $\underline{\text { alizado+a+15+de+abril++de+2016.pdf/a32b1dcf-7a4e-8a37-ac16-c121928719c8 }}$

Decreto 1295 de 1994 [Ministerio del Trabajo]. Por el cual se determina la organización y administración del Sistema General de Riesgos Profesionales. 24 de junio de 1994. http://www.secretariasenado.gov.co/senado/basedoc/decreto 1295 1994.html

Via Inveniendi Et Iudicandi

e-ISSN: 1909-0528 | DOI: https://doi.org/10.15332/19090528

Vol. 16 N.o 2 | julio-diciembre del 2021 
[Ministerio del Trabajo]. El Ministerio del Trabajo apoya al Sistema General de Riesgos Laborales para la reducción de la accidentalidad. 16 de marzo de 2021. https://www.mintrabajo.gov.co/prensa/comunicados/2021/marzo/el-ministeriodel-trabajo-apoya-al-sistema-general-de-riesgos-laborales-para-la-reduccion-de$\underline{\text { la- }}$ accidentalidad\#: :text=En\%20el\%20a\%C3\%B10\%202020\%20se\%20registraron\% 20450.110\%20accidentes\%20de\%20trabajo,2019\%20donde\%20se\%20presentaro $\underline{\mathrm{n} \% 20611.275}$

Feria, K. (2020). La seguridad y la salud en el trabajo. Una aproximación a través del derecho penal cubano. Revista IUSTA, 52, 1550. DOI: https://doi.org/10.15332/25005286.5481

Maldonado, G. (2017). La afiliación fraudulenta en el sistema de seguridad social integral colombiano. Via Inveniendi Et Iudicandi, 12(2), 73-99.

https://doi.org/10.15332/s1909-0528.2017.0002.04

Parra Osorio, L. Gaona Ramírez, J., Romero Pumalpa, D. y Acosta Fernández, M. (2019). Providencias favorables de acoso laboral y precariedad en altas cortes en Colombia. Abordaje fenomenològico. Revista Republicana, (26), 171-190. https://urepublicana.edu.co/ojs/index.php/revistarepublicana/article/view/520

Resolución 2400 de 1979 [Ministerio del Trabajo]. 22 de mayo de 1979. https://www.ilo.org/dyn/travail/docs/1509/industrial\%20safety\%20statute.pdf

Resolución 0312 de 2019 [Ministerio del Trabajo]. 13 de febrero de 2019. https://www.mintrabajo.gov.co/documents/20147/59995826/Resolucion+03122019-+Estandares+minimos+del+Sistema+de+la+Seguridad+y+Salud.pdf

Resolución 1016 de 1989 [Ministerio del Trabajo]. 31 de marzo de 1989. https://www.arlsura.com/images/stories/documentos/res 1016 1989.pdf

Sampieri, R. H., Fernández, C., Baptista, P. (2006). Metodología de la Investigación. McGraw-Hill.

Sánchez, D. A. (2015). Un nuevo concepto de culpa patronal. Universidad Externado de Colombia.

Yong, S. (2019). Introducción a la responsabilidad pública y privada. Ibáñez.

Via Inveniendi Et Iudicandi

e-ISSN: 1909-0528 | DOI: https://doi.org/10.15332/19090528

Vol. 16 N.० 2 | julio-diciembre del 2021 


\section{Anexo 1. Matriz de recolección de información y análisis documental}

\begin{tabular}{l|l|l|}
\multicolumn{1}{|c|}{ Variables } & Contenidos de las sentencias \\
\hline Número de la sentencia & \\
\hline Año &
\end{tabular}

Año

\begin{tabular}{l} 
Departamento/ciudad \\
\hline Magistrado ponente
\end{tabular}

\begin{tabular}{|l|l|}
\hline Resumen de los hechos & $\begin{array}{l}\text { ¿A quién le pasó? ¿Qué le pasó? ¿Qué tipo riesgo laboral } \\
\text { estaba presente? ¿Qué le lesión le ocurrió? ¿La empresa } \\
\text { investigó el accidente? ¿Cuáles fueron las causas? }\end{array}$ \\
\hline Resumen del problema jurídico & $\begin{array}{l}\text { ¿Cuál fue la razón de la decisión? (ratio decidendi) } \\
\text { ¿Cuáles fueron los dichos de paso? (obiter dicta) }\end{array}$ \\
\hline
\end{tabular}

\begin{tabular}{l} 
Consideraciones relevantes \\
$\begin{array}{l}\text { 1) ¿Hubo condena por daño emergente? } \\
\text { 2) ¿Hubo condena por lucro cesante? }\end{array}$ \\
3Hubo condena por daño moral? \\
\hline Estándares mínimos en gestión de los peligros y los riesgos (SG-SST)
\end{tabular}

Estándares mínimos en gestión de los peligros y los riesgos (SG-SST)

\begin{tabular}{l|l|l|l|l}
$\begin{array}{l}\text { Reporte, investigación y análisis de } \\
\text { los accidentes de trabajo. }\end{array}$ & ¿Se tuvo en cuenta? \\
\hline $\begin{array}{l}\text { Metodología para identificación de } \\
\text { peligros, evaluación y valoración de } \\
\text { riesgos }\end{array}$ & ¿Se tuvo en cuenta? \\
\hline $\begin{array}{l}\text { Identificación de peligros y } \\
\text { evaluación y valoración de riesgos } \\
\text { con participación de todos los } \\
\text { niveles de la empresa }\end{array}$ & ¿Se tuvo en cuenta? \\
\hline $\begin{array}{l}\text { Medidas de prevención y control } \\
\text { frente a peligros y riesgos } \\
\text { identificados (eliminación, } \\
\text { sustitución, controles de ingeniería, } \\
\text { controles administrativos y } \\
\text { elementos de protección personal). }\end{array}$ & ¿Se tuvo en cuenta? \\
\hline $\begin{array}{l}\text { Aplicación de medidas de } \\
\text { prevención y control por parte de los } \\
\text { trabajadores. }\end{array}$ & ¿Se tuvo en cuenta? \\
\hline $\begin{array}{l}\text { Procedimientos e instructivos } \\
\text { internos de seguridad y salud en el } \\
\text { trabajo }\end{array}$ & ¿Se tuvo en cuenta? \\
\hline $\begin{array}{l}\text { Inspecciones a instalaciones, } \\
\text { maquinaria o equipos. }\end{array}$ & \\
\hline $\begin{array}{l}\text { Mantenimiento periódico de las } \\
\text { instalaciones, equipos, máquinas, y } \\
\text { herramientas. }\end{array}$ & ¿Se tuvo en cuenta? \\
\hline
\end{tabular}

Via Inveniendi Et Iudicandi

e-ISSN: 1909-0528 | DOI: https://doi.org/10.15332/19090528

Vol. 16 N.o 2 | julio-diciembre del 2021 


\begin{tabular}{l|l}
\multicolumn{1}{|c|}{ Variables } & \multicolumn{1}{c}{ Contenidos de las sentencias } \\
\hline $\begin{array}{l}\text { Entrega de los elementos de } \\
\text { protección personal y capacitación } \\
\text { para el uso adecuado. }\end{array}$ & ¿Se tuvo en cuenta? \\
\hline
\end{tabular}

Método de investigación del

Accidente de Trabajo (enfoque

multicausal) causas inmediatas

(actos y condiciones inseguros),

causas básicas (factores personales

y factores de trabajo) y factores de

control

¿Se tuvo en cuenta?

Fuente: tomado y adaptado de Parra, Gaona, Romero y Acosta (2019).

Via Inveniendi Et Iudicandi

e-ISSN: 1909-0528 | DOI: https://doi.org/10.15332/19090528

Vol. 16 N.0 2 | julio-diciembre del 2021 